\title{
Controle de tráfego agrícola e atributos físicos do solo em área cultivada com cana-de-açúcar
}

\author{
Antoniane Arantes de Oliveira Roque ${ }^{(1)}$, Zigomar Menezes de Souza(1), Ronny Sobreira Barbosa ${ }^{(1)}$ \\ e Gustavo Soares de Souza(1)
}

\begin{abstract}
(1) Universidade Estadual de Campinas, Faculdade de Engenharia Agrícola, Departamento de Água e Solos, Avenida Candido Rondon, no 501, CEP 13083-875 Campinas, SP. E-mail: antoniane@yahoo.com.br, zigomarms@feagri.unicamp.br, ronny.barbosa@feagri.unicamp.br, gustavo.souza@feagri.unicamp.br
\end{abstract}

Resumo - O objetivo deste trabalho foi avaliar o efeito do controle de tráfego agrícola na compactação do solo em áreas cultivadas com cana-de-açúcar no sistema de colheita mecanizada. O trabalho foi realizado em delineamento de blocos ao acaso, em parcelas subsubdivididas com os tratamentos: colheita mecanizada tradicional; corte mecanizado com controle de tráfego; e corte mecanizado com controle de tráfego e uso do piloto automático. Os atributos físicos do solo foram avaliados nas camadas de $0,0-0,1,0,1-0,2$ e $0,2-$ $0,3 \mathrm{~m}$ de profundidade, na linha de plantio, no local em que o rodado passa e no entrerrodado. Avaliaram-se também os efeitos desses tratamentos sobre a produtividade da cana-de-açucar, respectivamente. O tráfego das máquinas agrícolas aumenta a densidade do solo e diminui o diâmetro médio ponderado dos agregados e a macroporosidade na linha de rodado em relação à linha de plantio, o que causa a degradação cumulativa da qualidade física do solo, ao longo dos anos de cultivo. Os dois anos de controle de tráfego agrícola da colheita não influenciaram os atributos físicos do solo estudados.

Termos para indexação: bitola, colheita mecânica, manejo do solo, piloto automático, trafegabilidade.

\section{Agricultural traffic control and soil physical attributes in sugarcane areas}

\begin{abstract}
The objective of this work was to evaluate the effect of traffic control on soil compaction in sugarcane areas with mechanical harvesting system. The study was carried out in a randomized complete block design in a split plot arrangement, with the following treatments: traditional mechanical harvesting, mechanical harvesting with traffic control, mechanical harvesting with traffic control and automatic tractor guidance. Soil physical properties were assessed at $0.0-0.1,0.1-0.2$ and $0.2-0.3-\mathrm{m}$ depths, at the planting row, at the wheel tracks, and between wheel tracks. The effects of these treaments on sugarcane yield were evaluated, respectively, too. Agricultural traffic increases soil density, and reduces aggregate mean geometric diameters and macroporosity at the wheel tracks in comparison to the planting row, which leads to cumulative soil physical quality degradation throughout the years. The two years of agricultural traffic control did not influence the studied soil physical attributes.
\end{abstract}

Index terms: gauge, mechanical harvesting, soil management, automatic tractor guidance, trafficability.

\section{Introdução}

O processo atual de produção de cana-de-açúcar está baseado em unidades onde se realizam o plantio e o cultivo em uma ou duas linhas, com tratores que têm no máximo 2,0 $\mathrm{m}$ de bitola. No entanto a colheita é feita em linhas simples, acompanhada do veículo de transbordo na linha adjacente. Como consequência, verifica-se um tráfego intenso, com baixo rendimento operacional, alto custo e elevada compactação dos solos. Esses são alguns dos fatores que obrigam a reimplantação do canavial a cada cinco anos.

Atualmente, com o incremento das áreas agrícolas, há maior preocupação com os problemas relacionados à compactação do solo resultante das operações mecanizadas, realizadas em condições de umidade elevadas (Silva \& Cabeda, 2006). O processo de compactação em um Latossolo Vermelho sob cultivo de cana-de-açúcar foi investigado por Paulino et al. (2004), que verificaram que o manejo de pós-colheita em soqueiras de cana-de-açúcar alterou a densidade do solo, a macro e a microporosidade. Porém, não observaram redução na área, no comprimento de raízes e na produção de cana-de-açúcar decorrentes do alto nível de compactação do solo. Souza et al. (2005), na avaliação de sistemas de colheita de cana queimada e crua com e sem incorporação da palhada de cana-de-açúcar, observaram que os sistemas de

Pesq. agropec. bras., Brasília, v.45, n.7, p.744-750, jul. 2010 
colheita alteraram os valores da densidade do solo, a macroporosidade e a estabilidade de agregados até a profundidade de $0,3 \mathrm{~m}$.

No contexto da agricultura mecanizada, o controle de tráfego surge como uma alternativa para o cultivo de cana-de-açúcar, pois minimiza os efeitos adversos da mecanização agrícola, separa as zonas de tráfego daquelas em que há crescimento das plantas e concentra a passagem de pneus em linhas delimitadas. Desse modo, uma área menor será submetida ao tráfego agrícola, embora mais intensamente (Laguë et al., 2003; Trein et al., 2005). Esta é uma prática recente no Brasil, mas bastante difundida na Austrália, que tem como objetivo reduzir o impacto da compactação do solo sobre a produtividade das culturas (Tullberg, 1997).

Em uma avaliação do efeito da posição do tráfego na colheita de cana-de-açúcar, Braunack et al. (2006) verificaram redução da compactação e aumento da produtividade da cultura em áreas com controle de tráfego. Os benefícios resultantes de menor compactação podem atingir também a produtividade e o ganho econômico do produtor. Pesquisas realizadas na Austrália detectaram aumento de $16 \%$ na produtividade e aumento de $30 \%$ nos lucros pelo uso do sistema de tráfego controlado quando comparado com o sistema convencional na produção de grãos de sorgo, trigo e milho (Tullberg, 1997).

A adoção do controle de tráfego permite melhorias na estrutura física do solo e redução no consumo de combustível, pois uma maior área de solo não será compactada e apresentará menor resistência à ruptura do solo na passagem de implementos de mobilização, bem como melhoria no potencial de tração do solo (relação pneu-solo), o que aumenta o rendimento da tração consequente do tráfego do maquinário em solo mais firme (linhas de tráfego).

O objetivo deste trabalho foi avaliar o efeito do controle de tráfego agrícola na compactação do solo em áreas cultivadas com cana-de-açúcar no sistema de colheita mecanizada de cana crua.

\section{Material e Métodos}

O experimento foi realizado na Usina São Martinho,

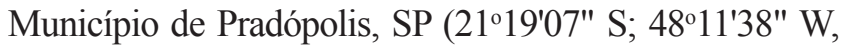
altitude de $630 \mathrm{~m})$. O clima é o mesotérmico de inverno seco (Cwa), pela classificação climática de Köppen. Aárea apresenta topografia plana e o solo é classificado como
Latossolo Vermelho distrófico típico álico, textura argilosa, A moderado (Santos et al., 2006).

A área do experimento foi plantada com cana-de-açúcar (Saccharum officinarum) em 2007, correspondente à renovação do canavial com a primeira colheita (cana planta ou primeiro corte), ocorrida em 2008 e, o segundo corte, em 2009. Foi realizada calagem na área, com $2,5 \mathrm{Mg} \mathrm{ha}^{-1}$ de calcário dolomítico a $32 \%$. A área recebeu adubação mineral com nitrato de amônio na quantidade de $310 \mathrm{~kg} \mathrm{ha}^{-1}$, e orgânica, com vinhaça, na quantidade de $100 \mathrm{~m}^{3} \mathrm{ha}^{-1}$, após o plantio. No plantio, foram ainda aplicados $20 \mathrm{Mg} \mathrm{ha}^{-1}$ de torta de filtro, utilizando-se plantadora modelo PCP, com peso de $8 \mathrm{Mg}$, distribuídos em quatro pneus de alta flutuação. A colhedora de cana-de-açúcar utilizada no experimento foi a de modelo 7700 (Case IH Agriculture, Piracicaba, Brasil), com 335 cv (246 kW) de potência bruta e $18,5 \mathrm{Mg}$ de peso, distribuídos em duas esteiras. Os transbordos, com peso médio de $8 \mathrm{Mg}$, distribuídos em quatro pneus de alta flutuação, tinham a capacidade para carregar até $10 \mathrm{Mg}$ de cana-de-açúcar. O trator utilizado na tração dos transbordos, modelo Magnum MX (Case IH Agriculture, Curitiba, Brasil), com $240 \mathrm{cv}(176 \mathrm{~kW})$ de potência, com 10,65 Mg de peso, distribuídos em dois pneus dianteiros $\mathrm{R} 30$ e dois pneus traseiros R38, ambos com pressão de inflação de $117 \mathrm{kPa}$.

A área experimental foi dividida em 12 parcelas, cada uma delas com 14 linhas de plantio com espaçamentos de $1,5 \mathrm{~m}$, o que totalizou $21 \mathrm{~m}$ de largura e $50 \mathrm{~m}$ de comprimento, com bordadura de $2,0 \mathrm{~m}$ no entorno da área. Os tratamentos em estudo foram: colheita mecanizada tradicional $(\mathrm{T})$; corte mecanizado com controle de tráfego e aumento de bitola para 3,0 m (CT); e corte mecanizado com controle de tráfego e uso do piloto automático (PA). Ocorreram duas passagens do maquinário entre as linhas da cultura na colheita, com uma frequência de seis passadas de trator no primeiro ano.

Foram coletadas amostras com e sem estrutura preservada, 40 dias após a colheita, para determinação dos atributos físicos do solo. Foram abertas trincheiras no sentido perpendicular às linhas de plantio, em que as amostras foram coletadas nas camadas de $0,0-0,1,0,1-0,2$ e $0,2-0,3 \mathrm{~m}$ de profundidade. Além da avaliação da compactação em profundidade, também foram feitas coletas em três diferentes pontos, tendo sido uma no local em que o rodado do trator deve 
trafegar, chamado de R, uma na linha de plantio (LP) e outra no entrerrodado (ER). Três trincheiras por parcela foram abertas aleatoriamente, com coleta de amostras em três profundidades em cada ponto de coleta.

$\mathrm{O}$ teor de matéria orgânica foi obtido pela metodologia de Claessen (1997). A estabilidade de agregados por via úmida foi obtida após pré-umedecimento das amostras de solo com álcool, em cada camada, e passagem por peneiras com abertura de malha de 4,76, 2,00, 1,00, 0,50, 0,25 e $0,12 \mathrm{~mm}$ e agitação lenta por $15 \mathrm{~min}$.

Amostrasdeestruturapreservadaforamcoletadascom cilindros volumétricos $(0,03 \times 0,05 \mathrm{~m})$ nas camadas em estudo, para determinação da micro e macroporosidade, com uso da mesa de tensão. A porosidade total e a densidade do solo foram determinadas de acordo com Claessen (1997). A resistência do solo à penetração foi avaliada nas três profundidades, com o penetrômetro de impacto modelo IAA/Planalsucar-Stolf, (Kamaq, Araras, Brasil) com ângulo de cone de $30^{\circ}$, e os resultados transformados segundo Stolf (1991). O teor de água no solo foi obtido segundo Claessen (1997) em todas as camadas estudadas.

O trabalho foirealizado emum experimento trifatorial (tratamentos $\mathrm{x}$ pontos de coleta $\mathrm{x}$ profundidade) com parcelas subdivididas no delineamento de blocos ao acaso com quatro repetições - subparcelas nos locais de amostragem e subsubparcelas em profundidade. Quanto à casualização, o fator tratamento foi casualizado nas parcelas principais, separadamente dentro de cada bloco, o fator ponto de coleta foi avaliado em cada subdivisão (subparcelas) de cada parcela principal (tratamentos), e o fator profundidade foi avaliado em profundidades diferentes em cada subparcela com três repetições (trincheiras), o que totalizou 324 observações. Os dados foram submetidos à análise de variância, de acordo com o teste $\mathrm{F}$, a 5\% de probabilidade e, no caso de existência de significância nas interações ou nos fatores principais, foi aplicado o teste de Tukey também, a 5\% de probabilidade, com uso do SAS (SAS Institute, 1999).

\section{Resultados e Discussão}

Constatou-se uma diminuição nos teores de matéria orgânica em profundidade, em 2008 e 2009 (Tabela 1). Essa redução mostrou-se mais acentuada da camada de $0,0-0,1$ para a camada de $0,2-0,3 \mathrm{~m}$, em razão da maior deposição superficial de resíduos vegetais, o que concorda com os resultados obtidos por De Bona et al. (2006). O teor de matéria orgânica apresentou diferença significativa entre os sistemas de manejo de cana-de-açúcar, com os maiores teores para o sistema de controle de tráfego e piloto automático. Os resultados obtidos concordam com os verificados por Souza et al. (2005), que observaram que o sistema de cana colhida mecanicamente apresenta aumento no teor de matéria orgânica do solo.

Não houve diferença significativa quanto à densidade e macroporosidade do solo nos sistemas de manejo estudados, em 2008 e 2009 (Tabela 1). O aumento da densidade do solo até a profundidade de $0,3 \mathrm{~m}$ deveu-se ao tráfego de máquinas pesadas durante as operações de plantio e colheita. Os valores de densidade do solo são coerentes com os observados por Hartemink (1998).

Nos diferentes sistemas de manejo avaliados em 2009, todos os tratamentos apresentaram valores de macroporosidade do solo, em todas as profundidades, inferiores a $0,10 \mathrm{~m}^{3} \mathrm{~m}^{-3}$ (Tabela 1), que é considerado um valor mínimo de porosidade de aeração necessário ao desenvolvimento do sistema radicular. Em 2008, foram verificados valores de macroporosidade próximos do limite crítico na linha de plantio e na região entrerrodados, os quais, em razão do tráfego de máquinas agrícolas, passaram à condição de críticos em 2009 (Tabela 2). Souza et al. (2006), ao avaliar a micromorfologia do solo e sua relação com atributos físicos e hídricos, em um Latossolo Vermelho eutroférrico, verificaram que o cultivo intensivo da cultura de cana-de-açúcar reduz significativamente a porosidade do solo, com maior evidência na macroporosidade.

Os resultados da análise da variância aplicada aos teores de água no solo não mostraram diferença significativa para os tratamentos e camadas estudadas em 2008 e 2009 (Tabela 1). Observa-se que os valores do teor de água no solo nas profundidades estudadas variaram entre 0,18 e $0,20 \mathrm{~g} \mathrm{~g}^{-1}$. Os resultados obtidos neste trabalho concordam com os de Souza et al. (2005), que verificaram menor valor de resistência do solo à penetração na camada $0,0-0,1 \mathrm{~m}$, em sistemas de colheita e manejo da palhada de cana-de-açúcar, em Latossolo Vermelho-Amarelo distrófico.

Independentemente do sistema de manejo da cana-de-açúcar, houve aumento da resistência do solo à penetração até $0,3 \mathrm{~m}$, o que confirma os dados de Silva 
et al. (2000), que avaliaram a resistência mecânica do solo à penetração pelo tráfego de uma colhedora em dois sistemas de manejo do solo, em Latossolo Vermelho. Observou-se aumento da resistência à penetração da profundidade de $0,1-0,2$ para $0,2-0,3 \mathrm{~m}$, com valores considerados restritivos ao desenvolvimento radicular (Tabela 1). Segundo Arshad et al. (1996), valores entre 2,0 e 4,0 MPa, em sistema de plantio direto, dificultam o desenvolvimento radicular das culturas. Dexter (1987) afirmou que a compactação do solo é mais prejudicial em solo seco, e que em condições de maior conteúdo de água pode haver crescimento radicular em valores de resistência do solo à penetração superiores a 4,0 MPa.

Os diferentes sistemas de manejo da cana-de-açúcar não apresentaram diferença quanto ao diâmetro médio ponderado dos agregados (DMP), porém verificou-se diferença em profundidade, em 2008 e 2009 (Tabela 1). Em diferentes sistemas de colheita da cana-de-açúcar, Souza et al. (2005) encontraram alterações nos atributos físicos. Esses autores verificaram, após cinco anos, maior DMP no sistema com cana crua, em relação ao de cana queimada. Houve redução da estabilidade de agregados em profundidade, que provavelmente está relacionada ao baixo teor de matéria orgânica (Assis \& Lanças, 2005).

Os teores de matéria orgânica foram mais baixos no solo onde passaram os rodados, para todos os sistemas de manejo da cana-de-açúcar estudados em 2008 e 2009 (Tabela 2). A movimentação e o preparo do solo para o cultivo favorecem as reações de oxidação da matéria orgânica, por meio do aumento da pressão parcial de oxigênio e da exposição de novas superfícies para o ataque microbiano. Sistemas mais conservacionistas, como o sistema de controle de tráfego e o piloto automático, tendem, com o tempo de manejo com cana crua, a apresentar um incremento de matéria orgânica na superfície do solo, com contribuição do acúmulo de palha na superfície do solo em razão da colheita mecanizada (Freixo et al., 2002).

Tabela 1. Atributos físicos do solo e teor de matéria orgânica para os tratamentos controle de tráfego, piloto automático e tradicional, em diferentes camadas do solo, em 2008 e $2009^{(1)}$.

\begin{tabular}{|c|c|c|c|c|c|c|}
\hline \multirow[t]{2}{*}{ Tratamento } & \multicolumn{3}{|c|}{2008} & \multicolumn{3}{|c|}{2009} \\
\hline & $0,0-0,1$ & $0,1-0,2$ & $0,2-0,3$ & $0,0-0,1$ & $0,1-0,2$ & $0,2-0,3$ \\
\hline & \multicolumn{6}{|c|}{ Densidade do solo $\left(\mathrm{kg} \mathrm{dm}^{-3}\right)$} \\
\hline Controle de tráfego & $1,05 \mathrm{Aa}$ & $1,14 \mathrm{Ab}$ & $1,21 \mathrm{Ab}$ & $1,25 \mathrm{Aa}$ & $1,28 \mathrm{Aa}$ & $1,27 \mathrm{Aa}$ \\
\hline Piloto automático & $1,09 \mathrm{Aa}$ & $1,14 \mathrm{Aab}$ & $1,19 \mathrm{Ab}$ & $1,26 \mathrm{Aa}$ & $1,22 \mathrm{Aa}$ & $1,25 \mathrm{Aa}$ \\
\hline \multirow[t]{2}{*}{ Tradicional } & $1,10 \mathrm{Aa}$ & $1,18 \mathrm{Ab}$ & $1,21 \mathrm{Ab}$ & $1,24 \mathrm{Aa}$ & $1,23 \mathrm{Aa}$ & $1,26 \mathrm{Aa}$ \\
\hline & \multicolumn{6}{|c|}{ Teor de água no solo $\left(\mathrm{g} \mathrm{g}^{-1}\right)$} \\
\hline Controle de tráfego & $0,18 \mathrm{Aa}$ & 0,19 Aa & $0,19 \mathrm{Aa}$ & $0,17 \mathrm{Aa}$ & $0,18 \mathrm{Aa}$ & $0,18 \mathrm{Aa}$ \\
\hline Piloto automático & $0,17 \mathrm{Aa}$ & $0,17 \mathrm{Aa}$ & $0,18 \mathrm{Aa}$ & $0,16 \mathrm{Aa}$ & $0,17 \mathrm{Aa}$ & $0,18 \mathrm{Aa}$ \\
\hline \multirow[t]{2}{*}{$\underline{\text { Tradicional }}$} & $0,19 \mathrm{Aa}$ & $0,20 \mathrm{Aa}$ & $0,20 \mathrm{Aa}$ & $0,18 \mathrm{Aa}$ & $0,18 \mathrm{Aa}$ & $0,18 \mathrm{Aa}$ \\
\hline & \multicolumn{6}{|c|}{ Teor de matéria orgânica $\left(\mathrm{g} \mathrm{kg}^{-1}\right)$} \\
\hline Controle de tráfego & $30,20 \mathrm{Aa}$ & $24,60 \mathrm{Aa}$ & $15,10 \mathrm{Ab}$ & $34,70 \mathrm{Aa}$ & $28,70 \mathrm{Aa}$ & $17,30 \mathrm{Ab}$ \\
\hline Piloto automático & $22,10 \mathrm{Aa}$ & $17,30 \mathrm{Aa}$ & $11,30 \mathrm{Ab}$ & $24,30 \mathrm{Aa}$ & $17,30 \mathrm{Aa}$ & $12,70 \mathrm{Ab}$ \\
\hline \multirow[t]{2}{*}{$\underline{\text { Tradicional }}$} & $17,70 \mathrm{Ba}$ & $12,20 \mathrm{Ba}$ & $9,30 \mathrm{Bb}$ & $21,00 \mathrm{Ba}$ & $15,00 \mathrm{Ba}$ & $10,30 \mathrm{Bb}$ \\
\hline & \multicolumn{6}{|c|}{ Macroporosidade $\left(\mathrm{m}^{3} \mathrm{~m}^{-3}\right)$} \\
\hline Controle de tráfego & $0,11 \mathrm{Aa}$ & $0,10 \mathrm{Aa}$ & $0,10 \mathrm{Aa}$ & $0,06 \mathrm{Aa}$ & $0,05 \mathrm{Aa}$ & $0,05 \mathrm{Aa}$ \\
\hline Piloto automático & $0,11 \mathrm{Aa}$ & 0,09Aa & $0,10 \mathrm{Aa}$ & $0,05 \mathrm{Aa}$ & $0,06 \mathrm{Aa}$ & $0,06 \mathrm{Aa}$ \\
\hline \multirow[t]{2}{*}{ Tradicional } & $0,11 \mathrm{Aa}$ & $0,08 \mathrm{Aa}$ & $0,10 \mathrm{Aa}$ & $0,04 \mathrm{Aa}$ & $0,05 \mathrm{Aa}$ & $0,05 \mathrm{Aa}$ \\
\hline & \multicolumn{6}{|c|}{ Resistência do solo à penetração (MPa) } \\
\hline Controle de tráfego & $1,31 \mathrm{Aa}$ & $2,54 \mathrm{Ab}$ & $2,76 \mathrm{Abc}$ & $1,25 \mathrm{Aa}$ & $3,52 \mathrm{Aab}$ & $5,06 \mathrm{Ab}$ \\
\hline Piloto automático & $1,46 \mathrm{Aa}$ & $2,74 \mathrm{Ab}$ & $3,06 \mathrm{Abc}$ & $1,89 \mathrm{Aa}$ & $3,63 \mathrm{Aab}$ & $4,70 \mathrm{Ab}$ \\
\hline \multirow[t]{2}{*}{$\underline{\text { Tradicional }}$} & $1,47 \mathrm{Aa}$ & $2,85 \mathrm{Ab}$ & $3,09 \mathrm{Abc}$ & $1,88 \mathrm{Aa}$ & $5,34 \mathrm{Ab}$ & $5,22 \mathrm{Ab}$ \\
\hline & \multicolumn{6}{|c|}{ Diâmetro médio ponderado de agregados (mm) } \\
\hline Controle de tráfego & $2,92 \mathrm{Aa}$ & $2,16 \mathrm{Ab}$ & $1,77 \mathrm{Ac}$ & $2,14 \mathrm{Aa}$ & $1,71 \mathrm{Ab}$ & $1,29 \mathrm{Ac}$ \\
\hline Piloto automático & $3,06 \mathrm{Aa}$ & $2,39 \mathrm{Ab}$ & $1,96 \mathrm{Ac}$ & $2,05 \mathrm{Aa}$ & $1,77 \mathrm{Aa}$ & $1,37 \mathrm{Ab}$ \\
\hline Tradicional & $3,02 \mathrm{Aa}$ & $2,36 \mathrm{Ab}$ & $1,77 \mathrm{Ac}$ & $2,08 \mathrm{Aa}$ & $1,90 \mathrm{Aa}$ & $1,37 \mathrm{Ab}$ \\
\hline
\end{tabular}


Em 2008 e 2009, os valores de densidade do solo e macroporosidade diferiram nos locais de coleta das amostras de solo (Tabela 2). Foram verificados, em 2008, maiores valores para a densidade do solo no rodado, que diferiu das posições da linha de plantio e entrerrodado.

Com apenas um ano de manejo, ocorreu aumento da densidade do solo na linha de plantio e em entrerrodado, o que demonstra que os sistemas de manejo com controle de tráfego e piloto automático não garantiram qualidade física - baixos valores de densidade do solo e resistência do solo à penetração e valores de macroporosidade acima de $0,1 \mathrm{~m}^{3} \mathrm{~m}^{-3}$ - nas regiões de maior desenvolvimento radicular da cultura de cana-de-açúcar (Tabela 2). Resultados semelhantes foram observados por Paulino et al. (2004), que avaliaram o efeito da escarificação de um Latossolo Vermelho em área cultivada com cana-de-açúcar e verificaram que o manejo de pós-colheita em soqueiras de cana-de-açúcar aumenta os valores da densidade do solo e da microporosidade e diminui o valor da macroporosidade.

Em 2008, os valores do diâmetro médio ponderado dos agregados (DMP) diminuíram em relação aos de 2009, com os menores valores observados em entrerrodados e rodados, que diferiram da linha de plantio (Tabela 2). Em todos os sistemas de manejo da cana-de-açúcar estudados, a estabilidade de agregados foi reduzida. Esse resultado também foi obtido por Góes et al. (2005), que observaram que o revolvimento do solo para o plantio e cultivo da cana-de-açúcar tem maiores efeitos na estabilidade de agregados do que a matéria orgânica e o tempo de cultivo, em Latossolo Vermelho distroférrico.

Em todos os tratamentos estudados, houve aumento da resistência do solo à penetração da linha de plantio para o rodado (Tabela 2), com valores considerados restritivos ao desenvolvimento radicular, o que também foi obtido por Lima et al. (2005), que avaliaram o tráfego de máquinas agrícolas em área cultivada com

Tabela 2. Atributos físicos do solo e teor de matéria orgânica para os tratamentos controle de tráfego, piloto automático e tradicional, na linha de plantio, no entrerrodado e no rodado do trator, em 2008 e 2009.

\begin{tabular}{|c|c|c|c|c|c|c|}
\hline \multirow[t]{2}{*}{ Tratamento } & \multicolumn{3}{|c|}{2008} & \multicolumn{3}{|c|}{2009} \\
\hline & Linha de plantio & Entrerrodado & Rodado & Linha de plantio & Entrerrodado & Rodado \\
\hline & \multicolumn{6}{|c|}{ Densidade do solo $\left(\mathrm{kg} \mathrm{dm}^{-3}\right)$} \\
\hline Controle de tráfego & $1,08 \mathrm{Aa}$ & $1,11 \mathrm{Aab}$ & $1,21 \mathrm{Ab}$ & $1,21 \mathrm{Aa}$ & $1,29 \mathrm{Aa}$ & $1,29 \mathrm{Aa}$ \\
\hline Piloto automático & $1,07 \mathrm{Aa}$ & $1,12 \mathrm{Aa}$ & $1,23 \mathrm{Ab}$ & $1,20 \mathrm{Aa}$ & $1,23 \mathrm{Aa}$ & $1,31 \mathrm{Aa}$ \\
\hline \multirow[t]{2}{*}{$\underline{\text { Tradicional }}$} & $1,08 \mathrm{Aa}$ & $1,16 \mathrm{Aab}$ & $1,25 \mathrm{Ab}$ & $1,16 \mathrm{Aa}$ & $1,29 \mathrm{Ab}$ & $1,28 \mathrm{Ab}$ \\
\hline & \multicolumn{6}{|c|}{ Teor de água no solo $\left(\mathrm{g} \mathrm{g}^{-1}\right)$} \\
\hline Controle de tráfego & $0,18 \mathrm{Aa}$ & $0,18 \mathrm{Aa}$ & $0,19 \mathrm{Aa}$ & $0,18 \mathrm{Aa}$ & $0,17 \mathrm{Aa}$ & 0,18 Aa \\
\hline Piloto automático & $0,18 \mathrm{Aa}$ & $0,17 \mathrm{Aa}$ & $0,17 \mathrm{Aa}$ & $0,17 \mathrm{Aa}$ & $0,17 \mathrm{Aa}$ & $0,17 \mathrm{Aa}$ \\
\hline \multirow[t]{2}{*}{ Tradicional } & $0,20 \mathrm{Aa}$ & $0,20 \mathrm{Aa}$ & $0,20 \mathrm{Aa}$ & $0,18 \mathrm{Aa}$ & $0,18 \mathrm{Aa}$ & $0,19 \mathrm{Aa}$ \\
\hline & \multicolumn{6}{|c|}{ Teor de matéria orgânica $\left(\mathrm{g} \mathrm{kg}^{-1}\right)$} \\
\hline Controle de tráfego & $34,00 \mathrm{Aa}$ & $27,00 \mathrm{Aa}$ & $19,70 \mathrm{Ab}$ & $34,00 \mathrm{Aa}$ & $27,00 \mathrm{Aa}$ & $19,70 \mathrm{Ab}$ \\
\hline Piloto automático & $19,30 \mathrm{Ba}$ & $19,30 \mathrm{Aa}$ & $15,70 \mathrm{Aa}$ & $19,30 \mathrm{Ba}$ & $19,30 \mathrm{Aa}$ & $15,70 \mathrm{Ab}$ \\
\hline \multirow[t]{2}{*}{ Tradicional } & $15,00 \mathrm{Ba}$ & $17,00 \mathrm{Ba}$ & $14,30 \mathrm{Aa}$ & $15,00 \mathrm{Ba}$ & $17,00 \mathrm{Ba}$ & $14,30 \mathrm{Aa}$ \\
\hline & \multicolumn{6}{|c|}{ Macroporosidade $\left(\mathrm{m}^{3} \mathrm{~m}^{-3}\right)$} \\
\hline Controle de tráfego & $0,11 \mathrm{Aa}$ & 0,11 Aa & $0,08 \mathrm{Ab}$ & $0,07 \mathrm{Aa}$ & $0,05 \mathrm{Aab}$ & $0,04 \mathrm{Ab}$ \\
\hline Piloto automático & $0,10 \mathrm{Aab}$ & $0,12 \mathrm{Aa}$ & $0,09 \mathrm{Ab}$ & $0,08 \mathrm{Aa}$ & $0,06 \mathrm{Aab}$ & $0,04 \mathrm{Ab}$ \\
\hline \multirow[t]{2}{*}{ Tradicional } & $0,10 \mathrm{Aab}$ & $0,11 \mathrm{Aa}$ & $0,08 \mathrm{Ab}$ & $0,07 \mathrm{Aa}$ & $0,03 \mathrm{Ab}$ & $0,04 \mathrm{Ab}$ \\
\hline & \multicolumn{6}{|c|}{ Resistência do solo à penetração (MPa) } \\
\hline Controle de tráfego & $1,50 \mathrm{Aa}$ & $3,43 \mathrm{Aa}$ & $4,91 \mathrm{Aa}$ & $1,35 \mathrm{Aa}$ & $2,25 \mathrm{Ab}$ & $3,00 \mathrm{Ac}$ \\
\hline Piloto automático & $1,30 \mathrm{Aa}$ & $2,95 \mathrm{Aa}$ & $5,97 \mathrm{Aa}$ & $1,64 \mathrm{Aa}$ & $2,49 \mathrm{Ab}$ & $3,14 \mathrm{Ac}$ \\
\hline \multirow[t]{2}{*}{$\underline{\text { Tradicional }}$} & $1,55 \mathrm{Aa}$ & $4,66 \mathrm{Aa}$ & $6,23 \mathrm{Aa}$ & $1,69 \mathrm{Aa}$ & $2,69 \mathrm{Ab}$ & $3,03 \mathrm{Ab}$ \\
\hline & \multicolumn{6}{|c|}{ Diâmetro médio ponderado de agregados $(\mathrm{mm})$} \\
\hline Controle de tráfego & $2,62 \mathrm{Aa}$ & $2,25 \mathrm{Aab}$ & $1,98 \mathrm{Ab}$ & $1,85 \mathrm{Aa}$ & $1,72 \mathrm{Aa}$ & $1,58 \mathrm{Aa}$ \\
\hline Piloto automático & $2,91 \mathrm{Aa}$ & $2,30 \mathrm{Ab}$ & $2,20 \mathrm{Ab}$ & $1,92 \mathrm{Aa}$ & $1,71 \mathrm{Aab}$ & $1,56 \mathrm{Ab}$ \\
\hline$\underline{T r a d i c i o n a l}$ & $2,68 \mathrm{Aa}$ & $2,29 \mathrm{Aa}$ & $2,19 \mathrm{Aa}$ & $2,04 \mathrm{Aa}$ & $1,67 \mathrm{Ab}$ & $1,64 \mathrm{Ab}$ \\
\hline
\end{tabular}


citros. A resistência do solo à penetração foi superior nos locais com maior movimentação dos veículos agrícolas, o que é coerente com o maior tráfego de máquinas e a maior densidade do solo, e corrobora estudos de Streck et al. (2004). Os valores do teor de água no solo estiveram muito próximos entre si, em todos os locais de amostragem, e comprometeu os resultados obtidos quanto à resistência do solo à penetração. Resultados semelhantes de resistência do solo à penetração foram observados por Souza et al. (2005), em área cultivada com cana crua, em Latossolo Vermelho-Amarelo distrófico.

Quanto à macroporosidade, observou-se que não houve diferença entre os locais de amostragem em 2008 (cana-soca) e, em 2009, esse valor diminuiu em todos os locais de amostragem, e o rodado diferiu do entrerrodado e da linha de plantio. Quanto à distribuição do tamanho dos poros, em 2009, os três locais de amostragem apresentaram valor abaixo do ideal e os locais de entrerrodados e rodados apresentaram valor de macroporosidade abaixo do valor mínimo necessário. Esse resultado evidencia o efeito do tráfego de máquinas pesadas nos tratos culturais e, principalmente, na colheita da cana-de-açúcar, o que corrobora resultados de Streck et al. (2004).

As produtividades da soqueira não apresentaram diferenças significativas entre os tratamentos, com média de 96,5 t ha-1 em 2008, mesmo que os atributos físicos do solo tenham mostrado diferenças entre os manejos e pontos de coletas estudados. Não se observou redução de produtividade entre os sistemas de manejo decorrente da compactação do solo, que em 2009 teve a média de 100,98 $\mathrm{t} \mathrm{ha}^{-1}$, pois o regime hídrico foi mais adequado ao desenvolvimento da cultura. Segundo Reinert et al. (2008), a restrição ao crescimento das raízes reduz de maneira acentuada a produtividade das culturas, em anos com ocorrência de deficit hídrico. Paulino et al. (2004) não observaram redução na área e no comprimento de raízes e na produção de cana-de-açúcar decorrentes do nível de compactação do solo, em soqueira de cana-de-açúcar, em Latossolo Vermelho.

\section{Conclusões}

1. O tráfego das máquinas agrícolas aumenta a densidade do solo, diminui o diâmetro médio ponderado e a macroporosidade na linha de rodado em relação à linha de plantio, o que causa a degradação cumulativa da qualidade física do solo, ao longo dos anos de cultivo.

2. Dois anos de controle de tráfego agrícola da colheita não influenciaram os atributos físicos do solo estudados.

\section{Referências}

ARSHAD, M.A.; LOWERY, B.; GROSSMAN, B. Physical tests for monitoring soil quality. In: DORAN, J.W.; JONES, A.J. (Ed.). Methods for assessing soil quality. Madison: Soil Science Society of America, 1996. p.123-141. (SSSA. Special publication, 49).

ASSIS, R.L. de; LANÇAS, K.P. Avaliação dos atributos físicos de um Nitossolo Vermelho distroférrico sob sistema plantio direto, preparo convencional e mata nativa. Revista Brasileira de Ciência do Solo, v.29, p.515-522, 2005.

BRAUNACK, M.V.; ARVIDSSON, J.; HAKANSSON, I. Effect of harvest traffic position on soil conditions and sugarcane (Saccharum officinarum) response to environmental conditions in Queensland, Australia. Soil and Tillage Research, v.89, p.103-121, 2006.

CLAESSEN, M.E.C. (Org.). Manual de métodos de análise de solo. 2.ed. Rio de Janeiro: Embrapa-CNPS, 1997. 212p.

DE BONA, F.D.; BAYER, C.; BERGAMASCHI, H.; DIECKOW, J. Carbono orgânico no solo em sistemas irrigados por aspersão sob plantio direto e preparo convencional. Revista Brasileira de Ciência do Solo, v.30, p.921-920, 2006.

DEXTER, A.R. Mechanics of root growth. Plant and Soil, v.98, p.303-312, 1987.

FREIXO, A.A.; MACHADO, P.L.O.A.; GUIMARÃES, C.M.; SILVA, C.A.; FADIGAS, F.S. Estoques de carbono e nitrogênio e distribuição de frações orgânicas de Latossolo do Cerrado sob diferentes sistemas de cultivo. Revista Brasileira de Ciência do Solo, v.26, p.425-434, 2002.

GÓES, G.B. de; GREGGIO, T.C.; CENTURION, J.F.; BEUTLER, A.N.; ANDRIOLI, I. Efeito do cultivo da cana-de-açúcar na estabilidade de agregados e na condutividade hidráulica do solo. Irriga, v.10, p.116-122, 2005.

HARTEMINK, A.E. Soil chemical and physical properties as indicators of sustainable land management under sugar cane in Papua New Guinea. Geoderma, v.85, p.283-306, 1998.

LAGUË, C.; AGNEW, J.; KHELIFI, M. Theoretical evaluation on the feasibility of controlled-traffic farming (CTF) using wide-span implement carriers (WSIC) for Canadian agriculture. In: ANUAL MEETING OF THE CSAE/SCGR, 2003, Montréal. Proceedings. Montréal: CSAE/SCGR, 2003. (CSAE. Paper, 03-233).

LIMA, H.V. de; LIMA, C.L.R. de; LEÃO, T.P.; COOPER, M.; SILVA, A.P. da; ROMERO, R.E. Tráfego de máquinas agrícolas e alterações de bioporos em área sob pomar de laranja. Revista Brasileira de Ciência do Solo, v.29, p.677-684, 2005.

PAULINO, A.F.; MEDINA, C.C.; AZEVEDO, M.C.B.; SILVEIRA, K.R.P.; TREVISAN, A.A.; MURATA, I.M. Escarificação de um Latossolo Vermelho na pós-colheita de 
soqueira de cana-de-açúcar. Revista Brasileira de Ciência do Solo, v.28, p.911-917, 2004.

REINERT, D.J.; ALBUQUERQUE, J.A.; REICHERT, J.M.; AITA, C.; ANDRADA, M.M.C. Limites críticos de densidade do solo para o crescimento de raízes de plantas de cobertura em Argissolo Vermelho. Revista Brasileira de Ciência do Solo, v.32, p.1805-1816, 2008.

SANTOS, H.G. dos; JACOMINE, P.K.T.; ANJOS, L.H.C. dos; OLIVEIRA, V.A. de; OLIVEIRA, J.B. de; COELHO, M.R.; LUMBRERAS, J.F.; CUNHA, T.J.F. (Ed.). Sistema brasileiro de classificação de solos. 2.ed. Rio de Janeiro: Embrapa Solos, 2006. 306p.

SAS INSTITUTE. SAS/STAT: user's guide. Versão 8. Cary: SAS Institute, 1999.

SILVA, A.J.N. da; CABEDA, M.S.V. Compactação e compressibilidade do solo sob sistemas de manejo e níveis de umidade. Revista Brasileira de Ciência do Solo, v.30, p.921-930, 2006.

SILVA, V.R.; REINERT, D.J.; REICHERT, J.M. Susceptibilidade à compactação de um Latossolo Vermelho-Escuro e de um Podzólico
Vermelho-Amarelo. Revista Brasileira de Ciência do Solo, v.24, p.239-249, 2000.

SOUZA, Z.M. de; MARQUES JÚNIOR, J.; COOPER, M.; PEREIRA, G.T. Micromorfologia do solo e sua relação com atributos físicos e hídricos. Pesquisa Agropecuária Brasileira, v.41, p.487-492, 2006.

SOUZA, Z.M. de; PRADO, R. de M.; PAIXÃO, A.C.S.; CESARIN, L.G. Sistemas de colheita e manejo da palhada de cana-de-açúcar. Pesquisa Agropecuária Brasileira, v.40, p.271-278, 2005.

STOLF, R. Teoria e teste experimental de fórmulas de transformação dos dados de penetrômetro de impacto em resistência do solo. Revista Brasileira de Ciência do Solo, v.15, p.229-235, 1991.

STRECK, C.A.S.; REINERT, D.J.; REICHERT, J.M.; KAISER, D.R. Modificações em propriedades físicas com a compactação do solo causada pelo tráfego induzido de um trator em plantio direto. Ciência Rural, v.34, p.755-760, 2004.

TREIN, C.R.; LEVIEN, R.; SOUZA, L.F.C. Tráfego controlado: pneus e compactação. Cultivar Máquinas, v.41, p.22-25, 2005.

TULLBERG, J.N. Tractor-based systems for traffic control in Australia. Landwards, v.52, p.12-15, 1997

Recebido em 27 de abril de 2010 e aprovado em 26 de junho de 2010 